\title{
Hand Osteoarthritis: investigating Pain Effects of estrogen-containing therapy (HOPE-e): a protocol for a feasibility randomised placebo-controlled trial
}

Ioana R. Marian ${ }^{1 *}$ (D, Megan Goff ${ }^{2,3}$, Jennifer A. E. Williams ${ }^{2,3}$, Malvika Gulati ${ }^{2,4,5}$, Mae Chester-Jones ${ }^{1}$, Anne Francis ${ }^{3}$, Marion Watson ${ }^{3,6}$, Tonia L. Vincent ${ }^{2,4,5}$, Sue Woollacott ${ }^{2}$, Charles Mackworth-Young ${ }^{4}$, Victoria Glover ${ }^{7}$, Dominic Furniss ${ }^{2,5}$, Matthew Gardiner ${ }^{2,8}$, Sarah E. Lamb ${ }^{9,10}$, Katy Vincent ${ }^{11}$, Vicki S. Barber ${ }^{3}$, Joanna Black ${ }^{3}$, Susan J. Dutton ${ }^{1}$ and Fiona E. Watt $2,4,5,12,13$

\begin{abstract}
Background: Hand osteoarthritis $(\mathrm{OA})$ is a common condition, causing pain, stiffness and reduced quality of life. Incidence is higher amongst women, particularly around the age of the menopause. Whilst the relationship between sex hormones and OA has been studied in vitro, in epidemiological studies and in clinical trials of hormone replacement therapy (HRT), this study is the first to investigate the effect of estrogen-containing therapy on hand pain in post-menopausal women with symptomatic hand $\mathrm{OA}$ in a randomised study design.

Methods: This is a feasibility study of a double-blinded placebo-controlled intervention with 1:1 randomisation to either a combination of conjugated estrogens $0.45 \mathrm{mg}$ and bazedoxifene acetate $20 \mathrm{mg}$ (Duavive) or placebo. The target population is post-menopausal women with symptomatic hand OA, aiming to recruit 60-90 study participants. The primary objective is to assess the feasibility of a future fully powered randomised controlled trial (RCT). Participants will take the study medication for 24 weeks and be followed up for 28 weeks after randomisation. The primary outcomes used to determine feasibility are eligible participant identification rates and routes; recruitment, randomisation and retention rates of eligible participants; study medication compliance; and the likelihood of unintentional unblinding. Secondary outcomes include measures of hand pain, function, appearance and menopausal symptoms. An end of study questionnaire and focus groups will help to refine the final protocol for a full study.
\end{abstract}

\footnotetext{
* Correspondence: ioana.marian@csm.ox.ac.uk

'Oxford Clinical Trials Research Unit, Centre for Statistics in Medicine, Nuffield Department of Orthopaedics, Rheumatology, and Musculoskeletal Sciences (NDORMS), University of Oxford, Oxford OX3 7LD, UK

Full list of author information is available at the end of the article
}

(c) The Author(s). 2021 Open Access This article is licensed under a Creative Commons Attribution 4.0 International License, which permits use, sharing, adaptation, distribution and reproduction in any medium or format, as long as you give appropriate credit to the original author(s) and the source, provide a link to the Creative Commons licence, and indicate if changes were made. The images or other third party material in this article are included in the article's Creative Commons licence, unless indicated otherwise in a credit line to the material. If material is not included in the article's Creative Commons licence and your intended use is not permitted by statutory regulation or exceeds the permitted use, you will need to obtain permission directly from the copyright holder. To view a copy of this licence, visit http://creativecommons.org/licenses/by/4.0/. The Creative Commons Public Domain Dedication waiver (http://creativecommons.org/publicdomain/zero/1.0/) applies to the data made available in this article, unless otherwise stated in a credit line to the data. 
Discussion: Identifying new treatments for symptomatic hand OA is a recognised research priority. The study will help us to understand whether there are sufficient interested and eligible individuals in this target population who would consider HRT for their hand symptoms. It will provide proof-of-concept RCT data on the effects of HRT on hand pain and other clinically relevant outcomes in this population. The study will gain valuable information on the feasibility of a full RCT and how best to run this. The findings will be published in a peer-reviewed journal and presented at a relevant conference.

Trial registration: ISRCTN12196200 registered on 15 January 2019.

Keywords: Hand osteoarthritis, Estrogen, Hormone replacement therapy, Clinical trial, Feasibility

\section{Background}

Hand osteoarthritis (OA) affects approximately 2 million people in the UK. The most common symptoms are hand pain, stiffness and functional difficulties. This can affect independence and the ability to work with associated negative health and social consequences $[1,2]$. Typically characterised by an early, painful inflammatory phase and bony remodelling, the condition may involve the interphalangeal joints of the thumb and the fingers (proximal and distal interphalangeal joints) and the base of the thumb [3]. Patients may have only interphalangeal joint involvement, only base of thumb involvement or both.

Recommendations for the management of hand OA include general advice on joint protection, hand exercises, splinting, analgesia (such as topical antiinflammatory gel) and sometimes intra-articular steroid injections (although their routine use is somewhat controversial) [4-6]. Whilst these treatments are often helpful, their effectiveness is frequently modest and they are not suitable for the entire population with hand OA [79]. Surgery remains an option in severe cases of hand OA [8]. Around $90 \%$ of those seeking specialist care for symptomatic hand OA are female [10]. Six hundred twenty thousand women aged 45-64 sought treatment for hand OA within UK primary care over a 7-year period [11]. The incidence of hand OA is higher in women than men at all ages; however the greatest relative risk compared with men is at the age of 50-55 years, which is around the age of menopause, a time of hormonal change and progressive estrogen deficiency [12-15].

There is evidence from a number of other sources that estrogens may be important in OA. Connective tissues including articular cartilage and bone have estrogen receptors and make aromatase, which synthesises estrogen in local tissues. Anti-estrogens such as aromatase inhibitors used to treat breast cancer can precipitate musculoskeletal pain or flares of symptomatic OA $[16,17]$. Times of low estrogen can be associated with pain vulnerability in women, potentially exacerbating pain in the context of disease $[18,19]$.
Women may take hormonal replacement therapy (HRT) to control menopausal symptoms such as hot flushes, sweats and gynaecological symptoms associated with estrogen deficiency. For women with an intact uterus, HRT formulations must include progestogens or selective estrogen receptor modulators (SERMs) in addition to estrogens; this is to reduce the risk of endometrial hyperplasia and cancer [20]. SERMs are a group of agents which bind to estrogen receptors [21]. The benefits of HRT need to be considered in the context of individual risk factors, particularly female cancers and thromboembolic disease [22, 23]. First time prescription of HRT to women who are more than 10 years postmenopause or over 65 years of age is inadvisable and may have accounted for much of the morbidity in earlier studies, as reflected in the current prescribing guidelines [24-27].

To date, there have been no randomised controlled trials (RCTs) or proof-of-concept studies testing HRT in individuals with symptomatic hand OA. Observational HRT studies using varying combinations of hormones give conflicting results, perhaps because of confounding factors in those with musculoskeletal symptoms seeking or commencing HRT [13]. In post hoc analysis from large HRT clinical trials, there was evidence for a beneficial effect on musculoskeletal pain in those receiving conjugated equine estrogens (CEE), some of whom probably had OA [28]. Similarly, when studying hip and knee OA in HRT clinical trial data, modest protection from hip and also knee replacement were seen in those receiving CEE alone [29, 30]. Effects of HRT on hand OA were not specifically examined in these trials. However beneficial effects of HRT in hand OA are suggested by some epidemiological study data [31, 32].

SERMs could also have beneficial effects in OA [33]. Unlike 'standard' HRT, which tends to give conflicting results, consistent improvements have been reported with SERMs in animal models of OA [33-35]. In a single reported clinical trial, raloxifene improved back and knee pain, some of which was attributable to OA [36]. Newer SERMs such as bazedoxifene have a better safety profile than early-generation SERMs such as raloxifene. 
However, unwanted effects such as worsening postmenopausal flushing still limit acceptability of SERMs as lone agents.

Aiming to mitigate the issues of either compound used alone, Duavive was developed by Pfizer as a first-in-class combination of CEE and bazedoxifene. Duavive was approved in the US and the EU in 2014 for symptoms of estrogen deficiency. It reduced hot flushes and improved menopausal quality of life with a good safety profile [37, 38]. This combination showed no significant breast or endometrial risk compared with placebo after 2 years in several large phase III trials [39].

We aim to test the hypothesis that Duavive can improve average hand pain in post-menopausal women with symptomatic hand OA. We will not test the individual components (estrogen and bazedoxifene) in view of the safety and tolerability considerations outlined above. The combination agent (or matched placebo) would be used here in all women, irrespective of uterine status, given the mechanistic hypothesis that both components (estrogen and bazedoxifene) may have effects on OA hand pain and disease. It is also possible that this estrogen-bazedoxifene combination may be more acceptable and more effective than either treatment alone. Given a lack of precedent for RCTs in this area and uncertainties regarding recruitment, retention and proof-of concept data (including estimated effect size), a feasibility study was designed. The Hand Osteoarthritis: investigating Pain Effects of estrogen-containing therapy (HOPE-e) feasibility RCT aims to address these uncertainties and to inform the design of a future adequately powered multicentre randomised trial.

\section{Methods}

\section{Study design}

HOPE-e is a parallel group, double-blind randomised placebo-controlled interventional study to test the feasibility of using a licensed form of HRT (Duavive) for an alternative indication. The study examines the feasibility of a full trial, including recruitment; acceptability of randomisation; acceptability and tolerability of the medication and tests; the selection and acceptability of the proposed outcomes. Patient and public involvement (PPI) actively informed the rationale, design and development of protocol and patient facing materials of this study. Similarly, an end of study questionnaire and focus group delivered after week 28 will give study participants the opportunity to contribute to the refinement of the procedures for a future definitive trial.

\section{Primary objective}

To assess the feasibility of a future fully powered RCT of Duavive in post-menopausal women with hand OA based on the following outcomes:
- Eligible participant identification rates and routes

- Recruitment, randomisation and retention rates

- Compliance (participant reported)

- Likelihood of unblinding

\section{Secondary objectives}

To refine outcomes for the full study and generate proof-of concept data (including estimated effect size) on whether estrogen-containing therapy improves hand pain and other secondary outcomes in post-menopausal women with hand OA. Secondary outcomes will assess hand pain and function, menopause symptoms and joint appearance. Average hand pain will be recorded daily over the 14 days preceding each study visit, as well as the recalled average hand pain for the past 14 days at the study visit. Daily pain rating will either be by smart phone or by paper diary, depending on participant preference (see "Data collection methods" section). All outcome measures to address the primary and secondary objectives are listed in Table 1.

The protocol follows the Standard Protocol Items: Recommendations for Interventional Trials (SPIRIT) guidelines [50]. The SPIRIT checklist is provided as Supplementary Table 1 in the Additional files.

\section{Study setting}

This feasibility study will be undertaken at a small number of sites, including a range of primary and secondary care National Health Service (NHS) sites in the UK. Participants will also be recruited directly from the community, through participant identification centres and via a number of advertising methods. A list of the study sites can be provided on request from the HOPE-e study office.

\section{Eligibility criteria}

In order to be eligible for the study, patients must comply with all of the following:

- Able to give written informed consent

- Female aged 40-65 years old

- In those with an intact uterus: at least 12 months of spontaneous amenorrhea (without any menstrual bleeding in last 12 months) and last menstrual period not more than 10 years ago

- In those who have undergone hysterectomy or are/ were using an intrauterine contraceptive device with progesterone local therapy (such as Mirena ${ }^{\mathrm{TM}}$ ): follicle stimulating hormone (FSH) $\geq 30 \mathrm{mIU} / \mathrm{ml}$ ) on screening blood test and a history of menopausal symptoms in the last 1 to 10 years, in keeping with appropriate timing of menopausal status

- Hand pain, aching or stiffness on most days in the last 3 months and fulfils American College of 
Table 1 Outcome measures

\begin{tabular}{|c|c|c|c|}
\hline \multirow{2}{*}{$\begin{array}{l}\text { Primary objectives outcome } \\
\text { measures }\end{array}$} & \multicolumn{3}{|c|}{ Secondary objectives outcome measures } \\
\hline & Pain and function outcomes & Menopause symptoms & Joint appearance \\
\hline $\begin{array}{l}\text { - Rates of eligible participant } \\
\text { identification (screening period) } \\
\text { - Rates of recruitment/ } \\
\text { randomisation from different } \\
\text { sources (screening period) } \\
\text { - Retention rates (throughout } \\
\text { study) } \\
\text { - Study medication compliance } \\
\text { - Bang's Blinding Index } \\
\text { (likelihood of unblinding) [40] }{ }^{\text {a }}\end{array}$ & $\begin{array}{l}\text { - Average hand pain (rated over } 14 \\
\text { days preceding the study visit) } \\
\text { - Pain manikin-capturing pain in } \\
\text { other joints in the } 4 \text { weeks } \\
\text { preceding the study visit }{ }^{\mathrm{a}} \\
\text { - Functional Index for Hand } \\
\text { OsteoArthritis (FIHOA) } \\
\text { questionnaire }[41,42]^{\mathrm{b}} \\
\text { - Grip strength, by Jamar } \\
\text { dynamometer }[43]^{\mathrm{b}} \\
\text { - EQ-5D-5L questionnaire }[44,45]^{\mathrm{b}}\end{array}$ & $\begin{array}{l}\text { - Menopause-specific Quality of Life (MEN- } \\
\text { QOL) questionnaire (Intervention 1-month } \\
\text { recall version) }[38,46,47]^{\mathrm{b}} \\
\text { - Greene Climacteric Scale }[48]^{\mathrm{b}}\end{array}$ & $\begin{array}{l}\text { - Cosmesis score (single } \\
\text { question, Michigan Hand } \\
\text { Questionnaire) [49] } \\
\text { - Investigator-recorded ten- } \\
\text { der and swollen joint } \\
\text { counts }^{\text {a }} \\
\text { - Photographic recording of } \\
\text { swollen hand joints }^{\text {a }}\end{array}$ \\
\hline
\end{tabular}

${ }^{\mathrm{a}} \mathrm{See}$ Fig. 1 for collection time points

bSee "Questionnaires" in Table 2 for collection time points

'Proposed primary outcome for full trial

Rheumatology clinical diagnostic criteria for hand OA (3 or more of the following) [51]:

$\bigcirc$ Hard tissue enlargement of two or more of the following joints: 2nd or 3rd distal interphalangeal joints (DIPJ), 2nd or 3rd proximal interphalangeal joints, first carpometacarpal joints

$\bigcirc$ Hard tissue enlargement of two or more of the DIPJs

$\bigcirc$ Fewer than three swollen metacarpophalangeal joints

$\bigcirc$ Deformity of at least one of the joints listed in the first point

- Minimum of two painful hand joints of any type (interphalangeal joints (IPJ) or base of thumbs)

- Hand pain that has not responded adequately to National Institute for Health and Clinical Excellence (NICE) core guidance for the management of OA [27], including the use of paracetamol or nonsteroidal anti-inflammatory topical gel, except where there is contraindication or intolerance

- Average hand pain is reported as typically more than 4 out of 10 in severity, or average hand pain in the last 7 days of $4 / 10$ or more on a visual analogue scale

- Able and willing to comply with all study requirements

Participants may not enter the study if any of the following apply:

- Other cause of hand pain, including inflammatory arthritis, connective tissue disorder, chronic pain, or alternative clinical diagnosis such as tenosynovitis or carpal tunnel syndrome

- Pregnancy or breast feeding, or risk of this during study

- Use of one or more prohibited treatments within specified timeframe, or not willing to avoid treatment for the duration of the study (see additional details in Supplementary Table 2)

- Presence of one or more medical contraindications to the use of systemic hormonal replacement therapy (see additional details in Supplementary Table 2)

- Any other significant or uncontrolled disease or disorder which may either put the participants at risk because of taking part in the study, or may influence the result of the study, or the participants' ability to participate

- Participants involved in another research trial involving an investigational product in the past 8 weeks

\section{Blood and urine testing}

Blood samples will be taken at screening as part of the eligibility process and at weeks 4,12 , and 24 for monitoring the safety of therapy. A mid-stream urine sample will be tested by dipstick for blood, protein and glucose at screening. The urine sample will be used to perform beta human chorionic gonadotropin $(\beta-\mathrm{HCG})$ testing by test stick to exclude pregnancy, at the investigator's discretion. For safety reasons, at all other visits, a urine dipstick for blood, protein, and glucose or urinary $\beta$-HCG testing can be performed at the discretion of the investigator.

\section{Pregnancy and contraception}

Duavive is only licensed for use in postmenopausal women and is contraindicated in women who are, or may become, pregnant. If pregnancy occurs during treatment, Duavive should be withdrawn immediately. Individuals entering the study must be at least 1 year after natural cessation of periods. A urinary pregnancy test will be conducted at the screening visit and subsequent visits at the investigator's discretion, for example in those who are within 2 years of final menstrual period, 
and/or under the age of 45 . Where there is clinical concern, those individuals will be advised to use two reliable methods of barrier contraception.

\section{Interventions}

In the intervention group, conjugated estrogens 0.45 mg-bazedoxifene acetate 20mg (Duavive, Pfizer) will be administered orally as a tablet once daily for 24 weeks. There is no possibility for dose modification during the study. A matched placebo was manufactured and packaged by MODEPHARMA Ltd., UK. The placebo and the study medication tablets are in blister packs and will be placed in opaque plain box packaging at the time of dispensing. Members of the research team and staff conducting participant visits will not handle the study medication, and participants will be asked to return unused medication to the local pharmacy for safe disposal.

Study participants, care providers, study team (except the statistician and one other member of the study team) and outcome assessors will be blinded to intervention.

\section{Participant timeline}

The timeline for participants is illustrated in Fig. 1.

\section{Recruitment}

Potentially eligible participants will be identified and approached via multiple routes in order to maximise recruitment. These include discussion at routine clinic appointments (primary care and different specialties in secondary care including rheumatology, orthopaedics, hand surgery and therapies); contacting individuals who have registered an interest in $\mathrm{OA}$ research; publicity leading to self-referral, both online (websites, Twitter and other social media, Short Message Service (SMS)) and offline (posters and flyers displayed in secondary care research sites, general practice surgeries and community spaces such as physiotherapy clinics and pharmacies, community magazine advertising). Following provision of a Participant Information Leaflet (PIL) to those expressing interest, key eligibility criteria will be assessed in those verbally consenting to an initial telephone pre-screen, following which individuals will be invited to attend a screening visit in person at the nearest recruitment site.

\section{Consent}

A medically qualified and GCP trained clinician will obtain informed consent from the patient at the screening visit at least $24 \mathrm{~h}$ after receiving the PIL, following patient verification of understanding of all aspects of the study and having answered all questions and documenting this by completion of the study informed consent form.

\section{Screening assessment}

Following consent, full eligibility, including that assessed by blood test results, will be reviewed as part of the screening assessment and prior to randomisation (see Fig. 1). An X-ray of both hands is also offered at the end of screening visit to all those who appear eligible and who have not undergone a hand X-ray in the last 3 years. Although the presence of radiographic hand OA is not required for inclusion in the study, its presence or absence will be reported for participants entering the study. Should an individual fail to meet eligibility criteria at screening assessment based on a reversible criterion (e.g. initiation of oral analgesia within 4 weeks of screening visit), re-screening may be carried out if the participant agrees. If a participant fails to meet the inclusion and exclusion criteria on review of results from the screening visit, they will be withdrawn and not proceed with the study. They will be contacted with an explanation and advice as appropriate.

\section{Randomisation}

Consenting, eligible participants will be randomised 1:1 to either Duavive or placebo at the baseline visit. Randomisation to the interventions will be carried out via a secure (encrypted) web-based randomisation service, the Oxford Clinical Trials Research Unit (OCTRU) Registration, Randomisation and Management of Product (RRAMP). Randomisation will be performed using a minimisation algorithm including a random element to ensure balanced allocation of participants across the two treatment groups, stratified by centre and the profile of painful joints (IPJ hand OA only; IPJ plus base of thumb OA; or base of thumb OA only) [52]. To prevent predictability, the minimisation algorithm will be seeded, randomising the first few participants using simple randomisation. Specific details will be stored within a randomisation and blinding plan, stored securely in the confidential statistical trial master file.

\section{Data collection methods}

Study outcome data will be collected during five face-toface study visits and one remote visit. Details of visit timings and outcomes are presented in Fig. 1 and Table 2; these are the following:

- Screening (eligibility)

- Baseline (randomisation, first prescription of study medication)

- 4 weeks (safety, adherence)

- 12 weeks (safety, adherence, second prescription of study medication)

- 24 weeks (primary outcome, advice on weaning study medication over subsequent 4 weeks) 


\section{PIL given and study explained}

Patient given time to decide whether they would like to enter the study

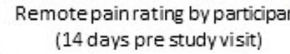

Randomisation

- Hand pain/global 0-10 scores/pain manikin

- Joint count/ joint function tests/Cosmesis score

- EQ-5D-5L/FIHOA/ MENQOL / Greene Climacteric scale

- Written informed consent obtained

- Eligibility assessed

- Demographics and physical examination

- Medical history taken

- Blood and urine screening

- Bilateral hand X-ray if required

- Training in remote pain reporting

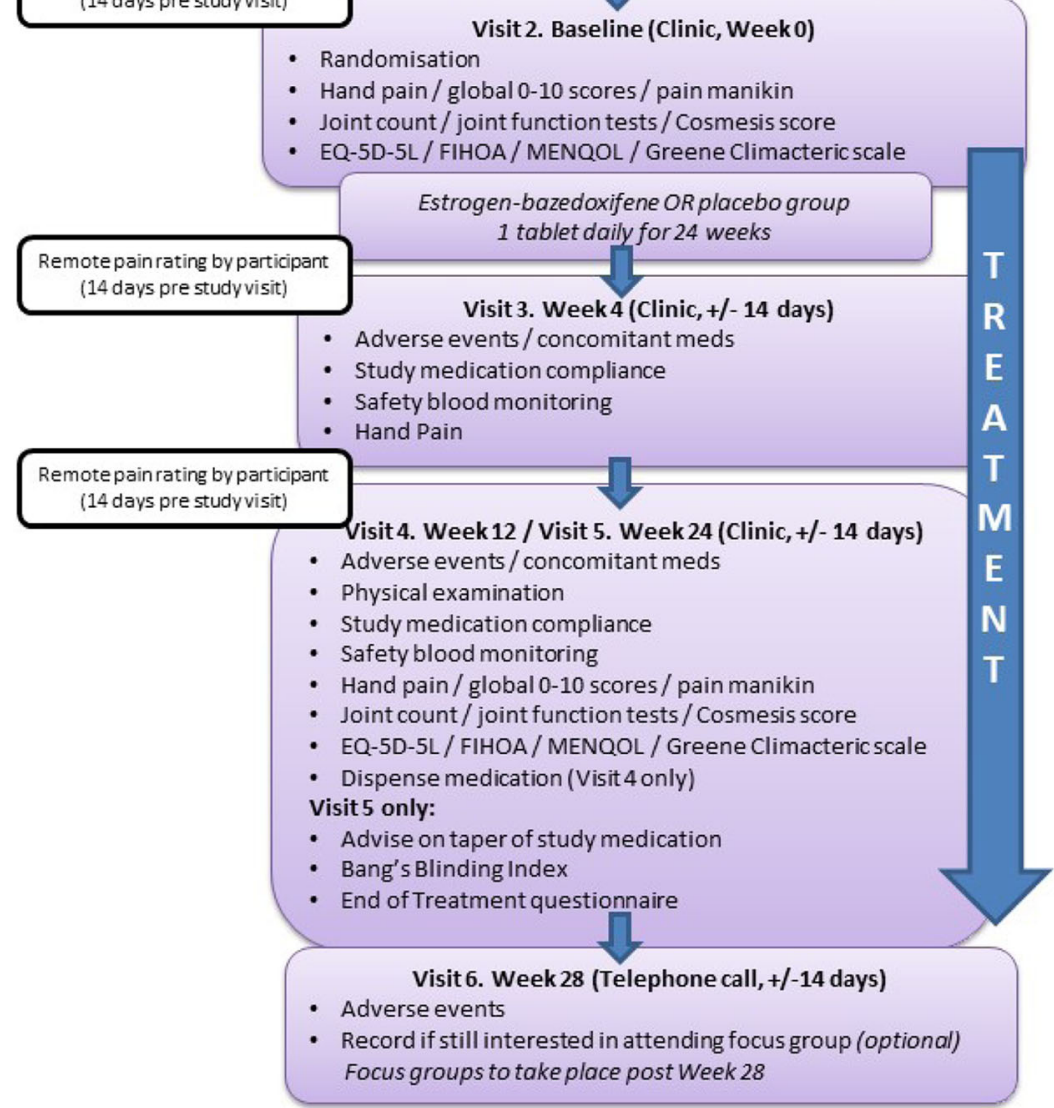

Fig. 1 Study flow chart

- 28 weeks phone call (safety, review of weaning of study medication)

We anticipate that any effects of the intervention on symptoms would be evident by between 4 and 24 weeks after initiation; therefore, the main outcomes will be collected at baseline, week 12 and week 24. Face-to-face visits will be necessary to assess for Adverse Events (AEs), provide new prescriptions and perform safety blood tests in addition to collection of other data (Fig. 1 and Table 2).
There will be a 28-week phone call to review progress following weaning of study medication after 24 weeks (best practice is to avoid abrupt cessation of HRT and this allows for this) and collect/assess any AEs. Some consenting participants will be randomly selected to attend one of two optional focus groups after week 28 .

Outcome data will be collected via Paper Case Report Forms completed by the investigator and participant. Remote daily rating of hand pain in the 14 days prior to a study visit will be captured on paper diaries or via the use of a single response online questionnaire 
Table 2 Timing of visits and data collection (SPIRIT)

\begin{tabular}{|c|c|c|c|c|c|c|}
\hline \multirow{4}{*}{ Procedures } & \multicolumn{6}{|l|}{ Visits/time points } \\
\hline & \multirow{3}{*}{$\begin{array}{l}1 \\
\text { Screening } \\
\text { Maximum } 42 \text { days }\end{array}$} & \multirow{2}{*}{$\begin{array}{l}2 \\
\text { Baseline }\end{array}$} & \multirow[t]{2}{*}{3} & \multirow[t]{2}{*}{4} & \multirow[t]{2}{*}{5} & \multirow{3}{*}{$\begin{array}{l}6 \\
\text { Follow-up } \\
\text { Week } 28\end{array}$} \\
\hline & & & & & & \\
\hline & & Week 0 & Week 4 & Week 12 & Week 24 & \\
\hline Informed consent & $x$ & & & & & \\
\hline Inclusion/exclusion & $x$ & $x$ & $x$ & $x$ & $x$ & \\
\hline Demographics & $x$ & & & & & \\
\hline Medical history & $x$ & & & & & \\
\hline Concomitant medication & $x$ & $x$ & $x$ & $x$ & $x$ & \\
\hline Hand pain 0-10 NRS & $x$ & $x$ & $x$ & $x$ & $x$ & \\
\hline Pain manikin & & $x$ & & $x$ & $x$ & \\
\hline Physical examination & $x$ & & $x$ & $x$ & $x$ & \\
\hline Vital signs & $x$ & & $x$ & $x$ & $x$ & \\
\hline Blood screening & $x$ & & & & & \\
\hline Urine dipstick & $x$ & $x^{a}$ & $x^{a}$ & $x^{a}$ & $x^{a}$ & \\
\hline Urine pregnancy test & $x^{a}$ & $x^{a}$ & $x^{a}$ & $x^{a}$ & $x^{a}$ & \\
\hline Bilateral hand X-ray (if applicable) & $x$ & & & & & \\
\hline Hand pain rating (remote) & Training & $x$ & $x$ & $x$ & $x$ & \\
\hline Randomisation & & $x$ & & & & \\
\hline Prescription/dispensing & & $x$ & & $x$ & & \\
\hline Reported study medication compliance & & & $x$ & $x$ & $x$ & \\
\hline Advice on barrier contraception & & $x^{a}$ & $x^{a}$ & $x^{a}$ & $x^{a}$ & \\
\hline Joint count & & $x$ & & $x$ & $x$ & \\
\hline Questionnaires & & $x$ & & $x$ & $x$ & \\
\hline Photographs of swollen hand joints & & $x$ & & $x$ & $x$ & \\
\hline Grip strength & & $x$ & & $x$ & $x$ & \\
\hline Safety blood monitoring ${ }^{c}$ & & & $x$ & $x$ & $x$ & \\
\hline Safety/adverse events & & & $x$ & $x$ & $x$ & $x$ \\
\hline Advice on weaning off study medication & & & & & $x$ & \\
\hline Bang's Blinding Index & & & & & $x$ & \\
\hline End of treatment questionnaire & & & & & $x$ & \\
\hline Focus group ${ }^{\mathrm{b}}$ (optional) & & & & & & \\
\hline
\end{tabular}

${ }^{\mathrm{a}}$ At discretion of investigator

${ }^{\mathrm{b}}$ After last participant has completed visit 6

'Includes creatinine, urea and electrolytes, liver function, full blood count and C-reactive protein

(Limesurvey) sent to the participant's smart phone via SMS, depending on the participant's preference.

\section{Data management}

All study data will be entered into a secure passwordprotected database (OpenClinica ${ }^{\circ}$ ), except for the electronic remote daily pain rating which is automatically stored in the study database. All hand photographs, audio recordings and transcripts of focus groups will be kept in a linked anonymised format and stored securely. Audio recordings will be deleted at the end of the study. A Data Management Plan which includes references to confidentiality, access and security arrangements has been produced for the study and is available on request from the HOPE-e study office.

\section{Concomitant medications}

Concomitant medication will be recorded at each visit. The participants will be immediately discontinued from the study medication if they commence any other systemic hormonal therapy (such as another form of systemic HRT or the oral contraceptive pill), any form of anti-estrogen medication or initiation of a liver-enzyme inducing agent. The medications or interventions 
described in Table 3 will be discouraged during the study; their use will be documented, but the participant will be allowed to remain in the study.

\section{Compliance with the study medication}

Clinically significant non-compliance will be defined as more than 14 days of missed medication in any calendar month, following which the participant will be withdrawn from study medication but invited to continue study follow-up.

\section{Breakthrough pain relief medication}

Participants may experience flares of hand pain during the study. During this time, they should take their usual analgesic medications as required. If possible, no new medications should be commenced for pain in the hand or elsewhere. Where paracetamol is not usually taken already, participants will be instructed that they can use paracetamol up to $1 \mathrm{~g}$ four times daily for the relief of breakthrough pain if they wish, preferably avoiding taking any in the $24 \mathrm{~h}$ prior to a study visit. Any use of such breakthrough pain relief will be documented at each visit, and the participant asked to record this in their study paper diary.

\section{Statistical analysis}

As this is a feasibility study not aiming to assess treatment effects but to test rates and feasibility of randomisation, a formal power calculation was not conducted. Instead we estimated 60-90 participants as the number required for the feasibility study in order to be able to accurately calculate a sample size that could detect a moderate standardised effect size of 0.3 to 0.5 in the future definitive trial [53]. Outcome data from this number of participants will be used to estimate standard deviations and confidence intervals (CIs) of the treatment estimates which will be used to inform a sample size calculation for a definitive trial.

The primary analysis will evaluate the feasibility of this study design based on the outcome measures for the primary research objectives described in Table 1: rates of

\section{Table 3 Concomitant medications}

\footnotetext{
- Intra-articular steroid, particularly when into the hands or within 3 months of the week 24 visit

- Oral steroid, particularly when for longer than 5 days consecutively or within 6 weeks of the week 24 visit

- Intramuscular steroid at any point during the study

- Use of intra-articular hyaluronan to a hand joint at any point during the study

- New prescription of non-steroidal anti-inflammatory drugs or other analgesic, particularly when within 4 weeks of the week 24 visit

- Initiation of treatment such as glucosamine or chondroitin at any point

- Initiation of hand exercises or other relevant non-pharmacological therapy at any point
}

eligible participants; rates of recruitment/randomisation; retention rates; and study medication compliance. These outcomes will be reported on an intention-to-treat (ITT) basis for each treatment group, with sensitivity analyses conducted on a per protocol (PP) basis. The PP population will exclude any participants with protocol deviations pre-specified in the analysis plan, such as reported non-compliance with the medication or reported concomitant medications use (as per Table 3). We will identify those who completed the study PP prior to unblinding of any analyses. Mean and standard deviation, or median and interquartile range, will be presented for continuous outcomes, depending on distribution, and numbers with percentages for binary and categorical data, for all planned primary and also for secondary outcome measures. Demographic and clinical characteristics for the two groups at baseline will be presented descriptively. Results will be reported descriptively as per Fig. 2 and in accordance with the Consolidated Standards of Reporting Trials (CONSORT) extension to randomised pilot and feasibility trials [54].

The secondary objective outcome measure, average hand pain is the planned primary outcome for the future definitive trial. This outcome is scored as $0-10$ on a $\mathrm{Nu}$ merical Rating Scale and collected from participant daily pain ratings for the 14 days prior to the baseline (week 0 ) and the week 24 study visit. The participant preference for hand pain data collection (online questionnaire or paper diary) will be compared and reported. Average hand pain will be reported as means and $95 \%$ CI based on a multivariate linear regression model with adjustment for the minimisation factors: study site (3 levels), and pattern of involvement of painful joints (IPJ hand OA only, IPJ plus base of thumb OA, or base of thumb OA only). This will be compared descriptively with the recalled average hand pain over the last 14 days at each visit, in terms of completion rates and differences between these measures. The change in average hand pain from baseline to 24 weeks and the difference in the means together and the corresponding 95\% CI will also be reported for each treatment group and overall. No formal statistical testing will be undertaken, as the study is not powered for this purpose. Additional analyses will include blood monitoring and safety data; pre-screen and screen-out rates; willingness to be randomised if eligible; and other measures of acceptability, including retention rates both overall and by recruitment site; response in those with IPJ hand OA only, IPJ and base of thumb OA, and base of thumb OA only; and those with or without evidence of radiographic hand OA. Further analysis outside of this primary analysis plan may be necessary to fully understand the feasibility of this study and to inform the future definitive trial. If this occurs, such analysis will be described in full in any results 


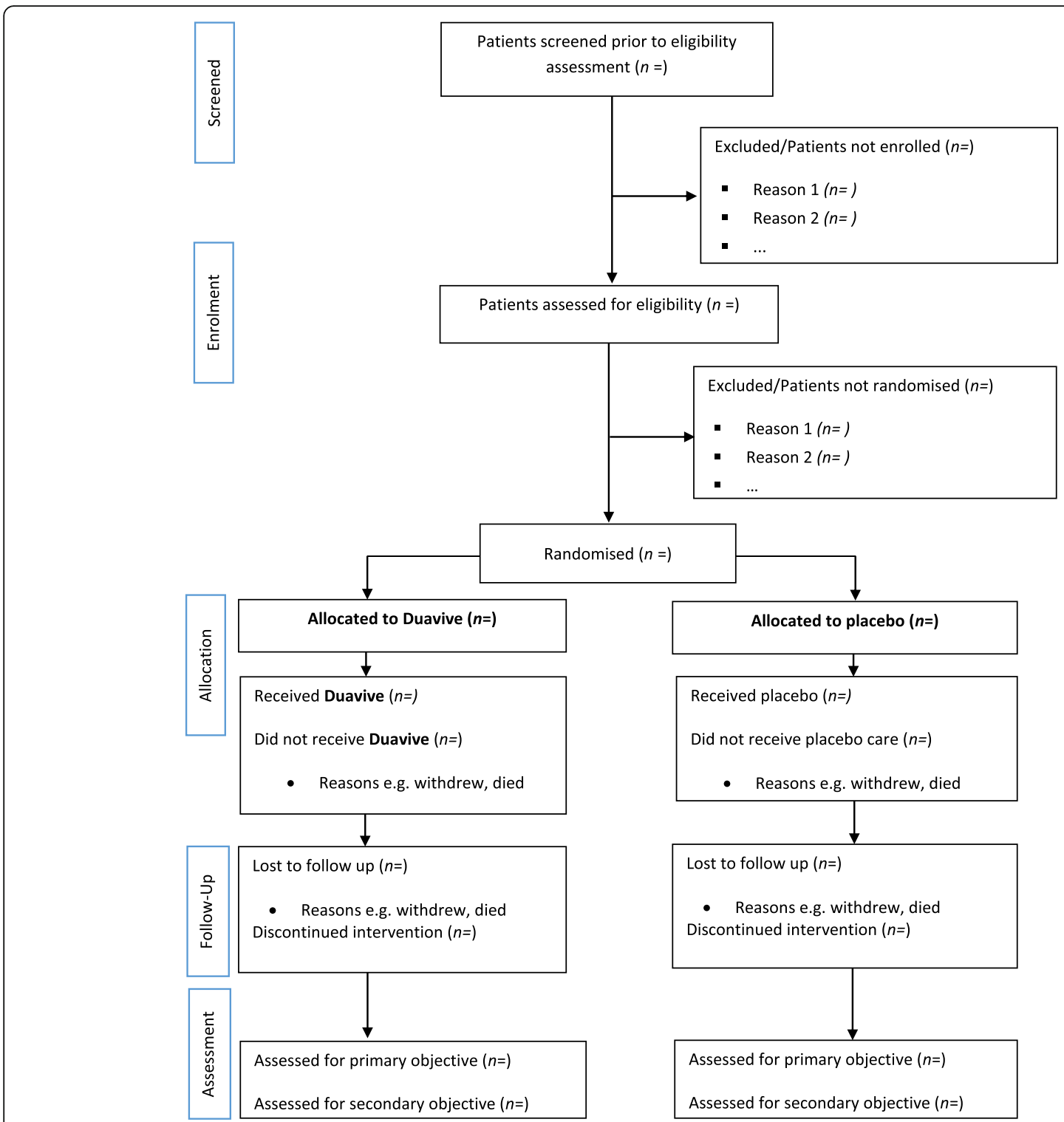

Fig. 2 CONSORT pilot and feasibility trials flow diagram schematic

publication arising from this feasibility study. Reasons for participant unblinding; missing data; withdrawals or loss to follow-up will be carefully considered and reported by treatment group and patterns of 'missingness' will be explored.

Data will be analysed using an appropriate validated statistical software such as Stata, StataCorp LP, USA [55]. No comparative interim analyses will be performed. A trial statistician will contribute to the statistical aspects of the study.

\section{Data monitoring}

A monitoring plan has been developed and will be followed throughout the study. A Trial Steering Committee (TSC) will oversee the overall conduct of the study and make recommendations on the feasibility of a full trial following completion. The TSC will comprise of invited expert members, including at least one medically qualified person, and patient representatives. A separate medically qualified Safety Oversight Clinician (SOC) (with appropriate clinical experience and independent to the study) will be appointed to safeguard the interest of the study participants. The SOC can advise the chair of the TSC at any time, if in their view, the study should be stopped due to concerns over participant safety.

Stop-go criteria for progression to a future definitive trial were predefined at the outset of the study. These were (i) recruitment of sufficient participants in a defined period (pre-specified as $\geq 30$ participants across all sites in 18 months, or, proportionate to this e.g. $\geq 22$ 
participants in 12 months); (ii) a drop-out rate of $\leq 30 \%$ of randomised individuals; and (iii) acceptability to the majority of participants, including acceptable rates of AEs. The TSC will make recommendations on the feasibility of a future definitive trial based in part, on all three of these pre-defined criteria being met.

\section{Withdrawals}

Participants have the right to withdraw from the study at any time and without giving reason. Participants asking to stop treatment or unable to tolerate the treatment will be withdrawn from the treatment. In the event of ineligibility (either arising during the study or retrospectively having been overlooked at screening) the participant will be withdrawn. The investigator may discontinue a participant from the study medication at any time if the investigator considers it necessary for any reason (e.g. unblinding; pregnancy; surgical procedures to the hand; AEs necessitating withdrawal of HRT; new diagnosis of cancer). Withdrawn participants will be given advice on weaning treatment if relevant, depending on the safety considerations. Those withdrawn from the study will be invited to an optional end of study visit at the time of their withdrawal.

\section{Safety}

All AEs will be collected at all visits as part of assessing the acceptability of the medication. These data will be summarised and reviewed by the SOC and the TSC. However, as this is a feasibility study with a licensed medication and an established safety profile, only rare events $(\geq 1 / 10,000$ to $<1 / 1000$, i.e. venous thromboembolic events including pulmonary embolism, retinal vein thrombosis, deep vein thrombosis and thrombophlebitis) and any other serious event deemed to be related and unexpected will be reported to the sponsor.

Worsening of the underlying disease as a direct result of the study medication will be reported as a Serious Adverse Reaction (SAR).

All serious adverse events (SAEs) will be reported to the trials unit within $24 \mathrm{~h}$ of the study team becoming aware. The relationship of each AE to the study medication will be determined by a medically qualified individual. These SAEs will also be reported to the Research Ethics Committee that gave a favourable opinion of the study if the chief investigator believes the event was 'related' (i.e. resulted from administration of any of the research procedures) and 'unexpected' in relation to those procedures.

In the event of a SAR or any suspicion of a SAR, the study medication will be stopped immediately. Stopping medication will also be considered for any AEs as part of the review process.
The following instances will also be reported as an $\mathrm{AE}$ and will need further investigation if they occur:

1. Those who experience postmenopausal bleeding and unblinding are carried out and they are shown to be on placebo.

2. Those who experience persistent or severe bleeding, ongoing bleeding beyond 12 weeks, or new bleeding after 12 weeks of taking Duavive (may represent a $\mathrm{SAE}$ ), depending on the underlying cause and the medical response to this.

When a participant has reported vaginal spotting or bleeding, they will be provided with a 'breakthrough bleeding diary' to record information on the nature and frequency of bleeding. Unblinding or withdrawal of study medication may be considered, and the advice of the study gynaecologist will be sought, particularly if further investigation is required. These events may represent a SAE, depending on the underlying cause. A trial specific instruction document has been designed to outline the review process in detail and is provided as Supplementary Figure 1 in Additional files.

\section{Unblinding}

Emergency and non-emergency unblinding will be available via the RRAMP system $24 \mathrm{~h}$ a day.

Accidental unblinding (for example due to breakthrough bleeding) of the participant or the investigator may occur and will be documented in an unblinding log, and its likelihood will be assessed at the end of the study. Accidental unblinding will not affect the majority of primary feasibility outcomes and participants would therefore be encouraged to continue their medication and complete the study. If a participant's allocation is unblinded for safety reasons during the study, the participant will be withdrawn from medication at that point but invited to attend follow-up study visits and complete the study.

\section{Auditing}

Direct access will be granted to authorised representatives from the sponsor, host institution and the regulatory authorities to permit study-related monitoring, audits and inspections.

\section{Ancillary and post-trial care}

There is no provision for continuation of the medication beyond the end of the study. If participants are eligible for HRT due to symptoms which are within standard indications for HRT use, the participant will be advised to discuss this with their GP. 


\section{Dissemination policy}

Reporting of the HOPE-e study will be in line with the CONSORT extension for randomised pilot and feasibility trials guidelines [54] and will be agreed at TSC meetings. The results of the study will be published and disseminated via oral reports at international meetings and on University of Oxford websites. Study results will be disseminated to study participants via letters following end of study and analysis.

\section{Protocol amendments}

Favourable opinion of two substantial amendments was obtained on 9 August 2019 and 18 December 2019. A history of the protocol amendments is presented in Supplementary Table 3.

\section{Confidentiality}

The study staff will ensure that the participants' confidentiality is maintained. The study will comply with the General Data Protection Regulation and Data Protection Act, which requires data to be anonymised as soon as it is practical to do so. Any communication containing participant identifiable data from sites will be by NHS. net email or secure encrypted fax. The participants will be identified only by a participant code on all study documents and study database. All documents will be stored securely and only accessible to authorised personnel.

\section{Declaration of interests}

Duavive was purchased through the Oxford University Hospitals NHS Foundation Trust Pharmacy Purchasing and Distribution Unit and distributed to sites. The selection of study medication was on academic grounds. Pfizer had no part in the conception, funding or provision of study medication for this study.

\section{Discussion}

There is an ongoing search to identify pharmacological therapies which improve pain or target underlying disease processes in $\mathrm{OA}$, slowing its progression. The preponderance of symptomatic $\mathrm{OA}$ in post-menopausal women, with a particularly close association between the age of menopause ( 50 years) and the incidence of hand OA, makes targeting estrogen-related pathways an attractive proposition in this disease.

Not all women can take HRT safely. Despite a large amount of supporting safety data since the Women's Health Initiative in appropriate age groups nearer to menopause, there remains distrust amongst some of the public, the media and parts of the medical profession about the use of HRT. Considering attitudes to medications in the acceptability and feasibility of this intervention is therefore critical, particularly in terms of the perceived risk and benefit of taking a medication in this target population.

The choice of agent in the study was important. Here, we have chosen a combined oral HRT: an estrogen and a SERM. By arguing mechanistically for both components, we have justified administration to all participants, irrespective of uterine status (usually those with hysterectomy would just take unopposed estrogen as HRT). We have selected an agent based on the preclin$\mathrm{ical} /$ clinical data supporting likelihood of effect (of both components), the reported lower incidence of breakthrough bleeding with this agent and because participants in our discussion group were attracted by this newer agent, especially as both components may have a positive effect. If an efficacy signal is seen (although not powered for this), this study would not demonstrate which element of the agent was mediating this. Subsequent mechanistic studies would be needed to deconvolute any effect.

Much of our evidence implicating the menopause in the onset of hand OA and for estrogen as a relevant target to date is circumstantial: from epidemiological studies, observational studies and convenience data from large HRT trials. There is further insight from a recently published report (since the development of our protocol) from electronic healthcare data in UK Community Practice Research Datalink [15]. This analysis emphasises the close temporal link to menopause (the highest rates of hand OA were seen in the year after menopause), but not all women have a temporal association to menopause (approximately $60 \%$ appear to). The study suggests that those starting HRT near to their menopause gained the most benefit in terms of protection from hand OA. In this protocol, to adhere to good practice in HRT prescribing, we mandate that participants will be at least 1 year after final menstrual period, and that they have established hand OA clinically. Whether this will prove too late, either in terms of the course of their menopause and/or their disease course to effect change on symptoms is yet to be determined. That cessation of HRT appears to be associated with onset of OA in this study is further evidence for a modulatory role for these agents, which needs to be better understood. It is important to note that here, the agent will be weaned gradually over 4 weeks, rather than being stopped abruptly to reduce the chances of any symptom flare.

To our knowledge, the study is unique, aiming to build our knowledge of whether it is possible to intervene in a poorly understood area, that of menopause and related female musculoskeletal health issues. It seeks to assess a potential tailored intervention for a group with high unmet clinical need. It will also give us valuable information on the best outcomes in this particular group of women with the condition. Particular strengths of the 
study are its testing of feasibility and acceptability of different ways of collecting average hand pain, which is an established outcome measure, although one that was highlighted by participants in our PPI as inadequately measured. HOPE-e is designed to determine whether a large $\mathrm{RCT}$ treating symptomatic hand $\mathrm{OA}$ in postmenopausal women with estrogen therapy is feasible. The study opened to recruitment in May 2019 (IRAS ID 236463) and is anticipated to close in February 2021.

\section{Abbreviations}

AE: Adverse events; $\beta$-HCG: Beta human chorionic gonadotropin;

CEE: Conjugated equine estrogens; Cl: Confidence interval;

CONSORT: Consolidated Standards or Reporting Trials; DIPJ: Distal interphalangeal joints; FIHOA: Functional Index for Hand OsteoArthritis;

FSH: Follicle stimulating hormone; GCP: Good Clinical Practice; HOPE-e: Hand Osteoarthritis: investigating Pain Effects in a randomised placebo-controlled feasibility study of estrogen-containing therapy; HRT: Hormone replacement therapy; IPJ: Interphalangeal joint; IT: Intention-to-treat;

MENQOL: Menopause-specific quality of life; NHS: National Health Service; NIHR: National Institute for Health Research; OA: Osteoarthritis; OCTRU: Oxford Clinical Trials Research Unit; PIL: Patient Information Leaflet; PP: Per protocol; PPI: Patient and public involvement; RRAMP: Registration, Randomisation and Management of Product; RCT: Randomised controlled trial; SAE: Serious adverse event; SAR: Serious adverse reaction; SERM: Selective estrogen receptor modulator; SMS: Short message service; SOC: Safety Oversight Clinician; SPIRIT: Standard Protocol Items: Recommendations for Interventional Trials; TSC: Trial Steering Committee; UK: United Kingdom; VTE: Venous thromboembolism

\section{Supplementary Information}

The online version contains supplementary material available at https://doi. org/10.1186/s40814-021-00869-1.

\section{Additional file 1: Supplementary Table 1. SPIRIT 2013 Checklist:}

Recommended items to address in a clinical trial protocol and related documents*. Supplementary Table 2. Eligibility Criteria (exclusion, further details). Supplementary Figure 1. Flow Chart for Postmenopausal Bleeding. Supplementary Table 3. Protocol Amendment History*.

\section{Acknowledgements}

We would like thank our participants, the members of the patient discussion group and other research team members (Susan Morris, Gretchen Brewer, Patrick Julier) involved in the day-to-day running of the study. This study will be conducted as part of the portfolio of trials in the UK Clinical Research Collaboration registered clinical trials unit-Oxford Clinical Trials Research Unit (OCTRU) at the University of Oxford. It will follow their Standard Operating Procedures ensuring compliance with the principles of Good Clinical Practice and the Declaration of Helsinki and any applicable regulatory requirements.

\section{Authors' contributions}

All authors contributed to the drafting of the manuscript and have read and approved the final manuscript. FEW is the chief investigator, protocol author and grant holder. SJD, IRM and MCJ participated in the statistical design of the study. KV contributed to gynaecology/safety aspects of the study. SW oversaw PPI for the study and is a lay co-applicant. All authors read and approved the final manuscript.

\section{Funding}

This paper presents independent research funded by the National Institute for Health Research (NIHR) under its Research for Patient Benefit Programme (Grant Reference Number PB-PG-0416-20023). The views expressed are those of the author(s) and not necessarily those of the NIHR or the Department of Health and Social Care. Additional support is received from the Oxford University Hospitals NHS Foundation Trust, local Clinical Research Network, the NIHR Oxford Biomedical Research Centre and Versus Arthritis through their support of the Centre for Osteoarthritis Pathogenesis Versus Arthritis (Grant 21621). The views expressed are those of the authors and not necessarily those of the NHS, the NIHR or the Department of Health. The University of Oxford is the sponsor of the study. The funders and the sponsor had no explicit role in the study design. FEW is supported by a UKRI Future Leaders Fellowship (Reference MR/S016538). She is also a member of Centre for Sport, Exercise and Osteoarthritis Research Versus Arthritis, University of Oxford (Grant 21595).

\section{Availability of data and materials}

Direct access to research data will be granted to authorised representatives of the sponsor, regulatory authorities or the host institution for monitoring and/or auditing of the study to ensure compliance with regulations.

\section{Declarations}

Ethics approval and consent to participate

This is a feasibility study of a study medication used outside of its licensed indication. This study is not a Clinical Trial of an Investigational Medicinal Product as defined by the EU Directive 2001/20/EC, given that the outcomes are related to feasibility rather than efficacy. The MHRA confirmed that as currently designed this feasibility study did not fall under their remit. A favourable ethical opinion has been obtained from the North of Scotland Research Ethics Service on 08 October 2018 (UKREC 18/NS/0100). Health Research Authority approval was obtained on 18 October 2018. The study has already undergone full external peer review as part of the ethics approval and funding process.

Written informed consent will be obtained from all participants prior to their participation in the study.

\section{Consent for publication}

Not applicable.

\section{Competing interests}

The authors declare they have no competing interests.

\section{Author details}

${ }^{1}$ Oxford Clinical Trials Research Unit, Centre for Statistics in Medicine, Nuffield Department of Orthopaedics, Rheumatology, and Musculoskeletal Sciences (NDORMS), University of Oxford, Oxford OX3 7LD, UK. ${ }^{2}$ Centre for Osteoarthritis Pathogenesis Versus Arthritis, Kennedy Institute of Rheumatology, NDORMS, University of Oxford, Oxford, UK. ${ }^{3}$ Oxford Clinical Trials Research Unit (OCTRU), Nuffield Department of Orthopaedics, Rheumatology, and Musculoskeletal Sciences (NDORMS) University of Oxford, Oxford OX3 7LD, UK. ${ }^{4}$ Rheumatology Department, Charing Cross Hospital, Imperial College Healthcare NHS Trust, London, UK. ${ }^{5}$ Nuffield Orthopaedic Centre, Oxford University Hospitals NHS Foundation Trust, Oxford, UK. ${ }^{6}$ Centre for Clinical Vaccinology and Tropical Medicine, University of Oxford, Churchill Hospital, Old Road, Oxford OX3 7LJ, UK. ${ }^{7}$ White Horse Medical Practice, Faringdon Medical Centre, Oxfordshire, UK. ${ }^{8}$ Wexham Park Hospital, Frimley Health NHS Foundation Trust, Frimley, UK. ${ }^{9}$ Centre for Rehabilitation Research, NDORMS, University of Oxford, Oxford, UK. ${ }^{10}$ College of Medicine and Health, Institute for Health Research, University of Exeter, St Luke's Campus, Heavitree Road, Exeter EX1 2LU, UK. ${ }^{11}$ Nuffield Department of Women's and Reproductive Health, University of Oxford, Oxford, UK. ${ }^{12}$ Centre for Sport, Exercise and OA Research Versus Arthritis, University of Oxford, Oxford, UK. ${ }^{13}$ Centre for Inflammatory Disease, Department of Immunology and Inflammation, Imperial College London, Commonwealth Building, Hammersmith Campus, Du Cane Road, London W12 0NN, UK.

Received: 28 July 2020 Accepted: 7 June 2021

Published online: 24 June 2021

\section{References}

1. Bijlsma JWJ, Berenbaum F, Lafeber FPJG. Osteoarthritis: An update with relevance for clinical practice. Lancet. 2011;377(9783):2115-26. https://doi. org/10.1016/S0140-6736(11)60243-2.

2. Zhang Y, Niu J, Kelly-Hayes M, Chaisson CE, Aliabadi P, Felson DT. Prevalence of symptomatic hand osteoarthritis and its impact on functional status among the elderly: The framingham study. Am J Epidemiol. 2002; 156(11):1021-7. https://doi.org/10.1093/aje/kwf141. 
3. Marshall M, Watt FE, Vincent TL, Dziedzic K. Hand osteoarthritis: clinical phenotypes, molecular mechanisms and disease management. Nat Rev Rheumatol. 2018;14(11):641-56. https://doi.org/10.1038/s41584-018-0095-4.

4. Kloppenburg M, Kroon FPB, Blanco FJ, Doherty M, Dziedzic KS, Greibrokk E, et al. 2018 update of the EULAR recommendations for the management of hand osteoarthritis. Ann Rheum Dis. 2019;78(1):16-24. https://doi.org/10.113 6/annrheumdis-2018-213826.

5. Heyworth BE, Lee JH, Kim PD, Lipton CB, Strauch RJ, Rosenwasser MP. Hylan versus corticosteroid versus placebo for treatment of basal joint arthritis: a prospective, randomized, double-blinded clinical trial. J Hand Surg Am. 2008;33(1):40-8. https://doi.org/10.1016/j.jhsa.2007.10.009.

6. Meenagh GK, Patton J, Kynes C, Wright GD. A randomised controlled trial of intra-articular corticosteroid injection of the carpometacarpal joint of the thumb in osteoarthritis. Ann Rheum Dis. 2004;63(10):1260-3. https://doi. org/10.1136/ard.2003.015438.

7. Kroon FPB, Rubio R, Schoones JW, Kloppenburg M. Intra-articular therapies in the treatment of hand osteoarthritis: a systematic literature review. Drugs Aging. 2016;33(2):119-33. https://doi.org/10.1007/s40266-015-0330-5.

8. Conaghan PG, Dickson J, Grant RL. Guidelines: Care and management of osteoarthritis in adults: Summary of NICE guidance. Bmj. 2008;336(7642): 502-3. https://doi.org/10.1136/bmj.39490.608009.AD.

9. Moe RH, Kjeken I, Uhlig T, Hagen KB. There is inadequate evidence to determine the effectiveness of nonpharmacological and nonsurgical interventions for hand osteoarthritis: an overview of high-quality systematic reviews. Phys Ther. 2009;89(12):1363-70. https://doi.org/10.2522/ptj.200803 98.

10. Punzi L, Ramonda R, Sfriso P. Erosive osteoarthritis. Best Pract Res Clin Rheumatol. 2004;18(5):739-58. https://doi.org/10.1016/j.berh.2004.05.010.

11. Arthritis Research UK. Osteoarthritis in General Practice: Data and Perspectives. University of Keele; 2013. https://www.versusarthritis.org/ media/2115/osteoarthritis-in-general-practice.pdf.

12. Oliveria SA, Felson DT, Reed J, Cirillo PA, Walker AM. Incidence of symptomatic hand, hip, and knee osteoarthritis among patients in a health maintenance organization. Arthritis Rheum. 1995;38(8):1134-41. https://doi. org/10.1002/art.1780380817.

13. Watt FE. Hand osteoarthritis, menopause and menopausal hormone therapy. Maturitas. 2016;83:13-8. https://doi.org/10.1016/j.maturitas.2015.09. 007.

14. Watt FE, Carlisle K, Kennedy D, Vincent TL. Menopause and hormone replacement therapy are important aetiological factors in hand osteoarthritis: results from a cross-sectional study in secondary care. Maturitas. 2015;81(1):128. https://doi.org/10.1016/j.maturitas.2015.02.092.

15. Burkard T, Rauch M, Spoendlin J, Prieto-Alhambra D, Jick SS, Meier CR. Risk of hand osteoarthritis in new users of hormone replacement therapy: a nested case-control analysis. Maturitas. 2020;132:17-23. https://doi.org/10.1 016/j.maturitas.2019.11.006

16. Burstein HJ. Aromatase inhibitor-associated arthralgia syndrome. Breast. 2007;16(3):223-34. https://doi.org/10.1016/j.breast.2007.01.011.

17. Castel LD, Hartmann KE, Mayer IA, Saville BR, Alvarez J, Boomershine CS, Abramson VG, Chakravarthy AB, Friedman DL, Cella DF. Time course of arthralgia among women initiating aromatase inhibitor therapy and a postmenopausal comparison group in a prospective cohort. Cancer. 2013; 119:2375-82. https://doi.org/10.1002/cncr.28016.

18. Smith YR, Stohler CS, Nichols TE, Bueller JA, Koeppe RA, Zubieta JK. Pronociceptive and antinociceptive effects of estradiol through endogenous opioid neurotransmission in women. J Neurosci. 2006;26(21):5777-85. https://doi.org/10.1523/JNEUROSCI.5223-05.2006.

19. Martin VT. Ovarian hormones and pain response: A review of clinical and basic science studies. Gend Med. 2009;6(PART 2):168-92.

20. Rossouw JE, Anderson GL, Prentice RL, LaCroix AZ, Kooperberg C, Stefanick $\mathrm{ML}$, et al. Risks and benefits of estrogen plus progestin in healthy postmenopausal women: Principal results from the women's health initiative randomized controlled trial. J Am Med Assoc. 2002;288(3):321-33. https://doi.org/10.1001/jama.288.3.321.

21. Komm BS, Mirkin S. An overview of current and emerging SERMs. J Steroid Biochem Mol Biol. 2014;143:207-22. https://doi.org/10.1016/j.jsbmb.2014.03. 003.

22. Davis SR, Lambrinoudaki I, Lumsden M, Mishra GD, Pal L, Rees M, et al. Menopause. Nat Rev Dis Prim. 2015;1(1):15004. https://doi.org/10.1038/ nrdp.2015.4
23. Type and timing of menopausal hormone therapy and breast cancer risk: individual participant meta-analysis of the worldwide epidemiological evidence. Lancet. 2019;394(10204):1159-68.

24. Utian $\mathrm{WH}$. A decade post $\mathrm{WHI}$, menopausal hormone therapy comes full circle - Need for independent commission. Climacteric. 2012;15(4):320-5. https://doi.org/10.3109/13697137.2012.678916.

25. Prentice RL. Postmenopausal hormone therapy and the risks of coronary heart disease, breast cancer, and stroke. Semin Reprod Med. 2014;32(6):41925. https://doi.org/10.1055/s-0034-1384624.

26. Stuenkel CA, Davis SR, Gompel A, Lumsden MA, Murad MH, Pinkerton JAV, et al. Treatment of symptoms of the menopause: An endocrine society clinical practice guideline. J Clin Endocrinol Metab. 2015;100(11):3975-4011. https://doi.org/10.1210/jc.2015-2236.

27. National Institute for Health and Care Excellence. Menopause: Diagnosis and Management. Nice. 2015;

28. Chlebowski RT, Cirillo DJ, Eaton CB, Stefanick ML, Pettinger M, Carbone LD, et al. Estrogen alone and joint symptoms in the Women's Health Initiative randomized trial. Menopause. 2018;25(11):1313-20. https://doi.org/10.1097/ GME.0000000000001235.

29. de Klerk BM, Schiphof D, Groeneveld FPMJ, Koes BW, van Osch GJVM, van Meurs JBJ, et al. Limited evidence for a protective effect of unopposed oestrogen therapy for osteoarthritis of the hip: A systematic review. Rheumatology. 2009;48(2):104-12. https://doi.org/10.1093/rheumatology/ ken390.

30. Cirillo DJ, Wallace RB, Wu L, Yood RA. Effect of hormone therapy on risk of hip and knee joint replacement in the women's health initiative. Arthritis Rheum. 2006;54(10):3194-204. https://doi.org/10.1002/art.22138.

31. Spector TD, Nandra D, Hart DJ, Doyle DV. Is hormone replacement therapy protective for hand and knee osteoarthritis in women?: The Chingford Study. Ann Rheum Dis. 1997;56(7):432-4. https://doi.org/10.1136/ard.56.7.432.

32. Maheu E, Dreiser RL, Guillou GB, Dewailly J. Hand osteoarthritis patients characteristics according to the existence of a hormone replacement therapy. Osteoarthr Cartil. 2000;8(SUPPL. A):S33-7.

33. Lugo L, Villalvilla A, Largo R, Herrero-Beaumont $G$, Roman-Blas JA. Selective estrogen receptor modulators (SERMs): New alternatives for osteoarthritis? Maturitas. 2014;77(4):380-4. https://doi.org/10.1016/j.maturitas.2014.01.016.

34. Sniekers YH, Weinans H, Bierma-Zeinstra SM, van Leeuwen JPTM, van Osch GJVM. Animal models for osteoarthritis: the effect of ovariectomy and estrogen treatment - a systematic approach. Osteoarthr Cartil. 2008;16(5): 533-41. https://doi.org/10.1016/j.joca.2008.01.002.

35. Christgau S, Tankó LB, Cloos PAC, Mouritzen U, Christiansen C, Delaissé JM, et al. Suppression of elevated cartilage turnover in postmenopausal women and in ovariectomized rats by estrogen and a selective estrogen-receptor modulator (SERM). Menopause. 2004;11(5):508-18. https://doi.org/10.1097/ 01.WCB.0000121484.18437.98.

36. Fujita T, Fujii Y, Munezane H, Ohue M, Takagi Y. Analgesic effect of raloxifene on back and knee pain in postmenopausal women with osteoporosis and/or osteoarthritis. J Bone Miner Metab. 2010;28(4):477-84. https://doi.org/10.1007/s00774-009-0155-6.

37. Lobo RA, Pinkerton JAV, Gass MLS, Dorin MH, Ronkin S, Pickar JH, et al. Evaluation of bazedoxifene/conjugated estrogens for the treatment of menopausal symptoms and effects on metabolic parameters and overall safety profile. Fertil Steril. 2009;92(3):1025-38. https://doi.org/10.1016/j. fertnstert.2009.03.113.

38. Abraham L, Pinkerton JV, Messig M, Ryan KA, Komm BS, Mirkin S. Menopause-specific quality of life across varying menopausal populations with conjugated estrogens/bazedoxifene. Maturitas. 2014;78(3):212-8. https://doi.org/10.1016/j.maturitas.2014.04.008.

39. Palacios S, Currie H, Mikkola TS, Dragon E. Perspective on prescribing conjugated estrogens/bazedoxifene for estrogen-deficiency symptoms of menopause: A practical guide. Maturitas. 2015;80(4):435-40. https://doi.org/1 0.1016/j.maturitas.2015.01.003.

40. Bang $H, \mathrm{Ni} L$, Davis CE. Assessment of blinding in clinical trials. Control Clin Trials. 2004;25(2):143-56. https://doi.org/10.1016/j.cct.2003.10.016.

41. Kloppenburg M, Maheu E, Kraus VB, Cicuttini F, Doherty M, Dreiser RL, et al. OARSI Clinical Trials Recommendations: design and conduct of clinical trials for hand osteoarthritis. Osteoarthr Cartil. 2015;23(5):772-86. https://doi.org/1 0.1016/j.joca.2015.03.007.

42. Dreiser RL, Maheu E, Guillou GB, Caspard H, Grouin JM. Validation of an algofunctional index for osteoarthritis of the hand. Rev Rhum. 1995:62(6 SUPPL. 1):43S-53S 
43. Mathiowetz V, Weber K, Volland G, Kashman N. Reliability and validity of grip and pinch strength evaluations. J Hand Surg Am. 1984;9(2):222-6. https://doi.org/10.1016/50363-5023(84)80146-X.

44. Van Hout B, Janssen MF, Feng YS, Kohlmann T, Busschbach J, Golicki D, et al. Interim scoring for the EQ-5D-5L: Mapping the EQ-5D-5L to EQ-5D-3L value sets. Value Heal. 2012;15(5):708-15. https://doi.org/10.1016/j.jval.2012. 02.008 .

45. Janssen MF, Pickard AS, Golicki D, Gudex C, Niewada M, Scalone L, et al. Measurement properties of the EQ-5D-5L compared to the EQ-5D-3L across eight patient groups: a multi-country study. Qual Life Res. 2013;22(7):171727. https://doi.org/10.1007/s11136-012-0322-4.

46. Lewis JE, Hilditch JR, Wong CJ. Further psychometric property development of the Menopause-Specific Quality of Life questionnaire and development of a modified version, MENQOL-Intervention questionnaire. Maturitas. 2005; 50(3):209-21. https://doi.org/10.1016/.maturitas.2004.06.015.

47. Radtke JV, Terhorst L, Cohen SM. The menopause-specific quality of life questionnaire: Psychometric evaluation among breast cancer survivors. Menopause. 2011;18(3):289-95. https://doi.org/10.1097/gme.0b013e3181 ef975a.

48. Greene JG. Constructing a standard climacteric scale. Maturitas. 1998;29(1): 25-31. https://doi.org/10.1016/S0378-5122(98)00025-5.

49. Chung KC, Pillsbury MS, Walters MR, Hayward RA. Reliability and validity testing of the Michigan Hand Outcomes Questionnaire. J Hand Surg Am. 1998:23(4):575-87. https://doi.org/10.1016/S0363-5023(98)80042-7.

50. Chan AW, Tetzlaff JM, Altman DG, Laupacis A, Gøtzsche PC, Krleža-Jerić K, et al. SPIRIT 2013 statement: Defining standard protocol items for clinical trials. Ann Inter Med. 2013;158(3):200-7. https://doi.org/10.7326/0003-4819-1 58-3-201302050-00583.

51. Altman R, Alarcon G, Appelrouth D, Bloch D, Borenstein D, Brandt K, et al. The American College of Rheumatology criteria for the classification and reporting of osteoarthritis of the hand. Arthritis Rheum. 1990;33(11):1601-10. https://doi.org/10.1002/art.1780331101.

52. Brown S, Thorpe H, Hawkins K, Brown J. Minimization - Reducing predictability for multi-centre trials whilst retaining balance within centre. Stat Med. 2005;24(24):3715-27. https://doi.org/10.1002/sim.2391.

53. Whitehead AL, Julious SA, Cooper CL, Campbell MJ. Estimating the sample size for a pilot randomised trial to minimise the overall trial sample size for the external pilot and main trial for a continuous outcome variable. Stat Methods Med Res. 2015;25(3):1057-73. https://doi.org/10.1177/096228021 5588241.

54. Eldridge SM, Chan CL, Campbell MJ, Bond CM, Hopewell S, Thabane L, et al. CONSORT 2010 statement: extension to randomised pilot and feasibility trials. BMJ. 2016;355:i5239. https://doi.org/10.1136/bmj.i5239.

55. StataCorp. Stata Statistical Software: Release 16. College Station: StataCorp LLC; 2019.

\section{Publisher's Note}

Springer Nature remains neutral with regard to jurisdictional claims in published maps and institutional affiliations.

Ready to submit your research? Choose BMC and benefit from:

- fast, convenient online submission

- thorough peer review by experienced researchers in your field

- rapid publication on acceptance

- support for research data, including large and complex data types

- gold Open Access which fosters wider collaboration and increased citations

- maximum visibility for your research: over $100 \mathrm{M}$ website views per year

At $\mathrm{BMC}$, research is always in progress.

Learn more biomedcentral.com/submissions 